OPEN ACCESS

Edited by:

Greta Vladeanu,

Xylem, United States

Reviewed by:

Sumit Purohit,

Pacific Northwest National Laboratory (DOE), United States

Thomas Chen,

Xylem, United States

*Correspondence:

Nelson Carriço

nelson.carrico@estbarreiro.ips.pt

Specialty section This article was submitted to Water and Built Environment,

a section of the journal

Frontiers in Water

Received: 21 February 2021 Accepted: 24 May 2021 Published: 05 July 2021

Citation:

Carriço N and Ferreira B (2021) Data and Information Systems

Management for the Urban Water Infrastructure Condition Assessment.

Front. Water 3:670550

doi: 10.3389/frwa.2021.670550

\section{Data and Information Systems Management for the Urban Water Infrastructure Condition Assessment}

\author{
Nelson Carriço* and Bruno Ferreira \\ Centre for Innovation in Science and Technology, Barreiro School of Technology, Polytechnic Institute of Setúbal, Setúbal, \\ Portugal
}

Most of urban water infrastructure around the world were built several decades ago and nowadays they are deteriorated. So, the assets that constitute these infrastructures need to be rehabilitated. Since most of the assets are buried, water utilities face the challenge of deciding how, where and when to rehabilitate. Condition assessment is a vital component on plan rehabilitation actions and is mostly based on the data collected from the managed networks. This collected data need to be put together in order to be transformed into useful information. Nonetheless, the large amount of assets and data involved makes data and information management a challenging task for water utilities, especially in those with as lower digital maturity level. This paper highlights the importance of data and information systems' management for urban water infrastructure condition assessment based on the authors' experience.

Keywords: condition assessment, data, information systems, management, urban water infrastructure

\section{INTRODUCTION}

Urban water infrastructures are constituted by a large variety of physical assets (e.g., tanks, pumps, pipes). These assets deteriorate due to its natural aging processes and non-controlled processes (e.g., pipe's poor-quality production, external actions such as works) and, as a result, need to be rehabilitated. Rehabilitation is thus the process of upgrading asset's condition to its "as-new" condition if practicable (WRc, 2001; IPWEA and NAMS, 2015). Therefore, it is vital to assess the physical condition and functionality of urban water infrastructures, allowing the estimation of the remaining service life and asset value (Feeney et al., 2009). Since urban water infrastructures assets are mostly buried, the assessment of its condition must be based on reliable pre-acquired information.

Over the last decades, water utilities have made significant investments in implementing different information systems to address the increasing complexity of the daily control, operation, management, and planning of their systems (Halfawy, 2008). Data is usually collected, stored, managed, and analyzed using various information systems which are often disperse in different divisions of a water utility. The activity of condition assessment is data intensive and uses a plethora of information systems (Haider, 2007).

This paper highlights the importance of data and information systems' management for the urban water infrastructure condition assessment based on the authors' experience obtained from some Portuguese R\&D projects. These projects aimed to develop a platform to assist small and medium sized water utilities with low digitalization maturity level to integrate data from their different existing information systems to assess the condition of their water distribution systems. 
TABLE 1 | Set of performance indicators to assess and prioritize WSS or DMA.

\begin{tabular}{ll}
\hline Designation & Unit \\
\hline Non-revenue water & $(\%)$ \\
Network Rehabilitation & $(\% /$ year $)$ \\
Pipe failure & {$[\# /(100 \mathrm{~km}$. year $)]$} \\
Real water losses in network & {$\left[\mathrm{m}^{3} /(\mathrm{km}\right.$. year $\left.)\right]$} \\
Real water losses in service connections & {$[$ liters/(service connection. day) $]$} \\
Energy efficiency of pumping installations & {$\left[\mathrm{kWh} /\left(\mathrm{m}^{3} .100 \mathrm{~m}\right)\right]$} \\
Energy in excess per unit of input volume & $\left(\mathrm{kWh} / \mathrm{m}^{3}\right)$ \\
Energy in excess per unit of the revenue & $\left(\mathrm{kWh} / \mathrm{m}^{3}\right)$ \\
water & $(-)$ \\
Ratio of the total energy in excess & $(\%)$ \\
Unmetered consumption & {$[\# /(1,000$ service connections. year $)]$} \\
Service connection failure & $($ days $)$ \\
Treated water volume capacity & {$[\mathrm{hour} /(100$ users. year $)]$} \\
Disruption caused by pipe failures & {$[\mathrm{hour} /(100$ users. year $)]$} \\
Disruption caused by service connection \\
failures & \\
Real water losses & $(\%)$ \\
Infrastructure value index & $(-)$
\end{tabular}

\section{URBAN WATER INFRASTRUCTURE CONDITION ASSESSMENT}

Condition assessment may be defined as the identification of the likelihood that an asset will continue to perform its required function (AWWA, 2019) and is an essential part of any urban water infrastructure asset management (IAM) process. Several concepts and methodologies of IAM may be found in the bibliography (BSI, 2008; Almeida and Cardoso, 2010; Ugarelli et al., 2010; Alegre and Coelho, 2012; Matthews et al., 2012; Osman, 2012; IAM, 2015; IPWEA and NAMS, 2015; Beuken et al., 2020). According to the ISO 55000, asset management is the coordinated activity carried by an organization to realize value from its assets involving a balancing of costs, risks, opportunities, and performance benefits (ISO, 2014). So, condition assessment may involve risk, performance, and cost methodologies.

The most common risk management framework used are in accordance with the reference standards AS/NZS 4360:2004 and ISO 31000:2018 (AS/NZS, 2004; ISO, 2018). Risk assessment includes the processes of risk identification, risk analysis and risk evaluation (ISO, 2009). A performance assessment system is the set of data, calculations, performance metrics, and contextual information that allow the evaluation and reporting the performance of a single asset, a whole infrastructure, a service provided or a utility (Alegre and Covas, 2010; Almeida and Cardoso, 2010). The cost assessment aims at evaluating: (i) the deterioration of an infrastructure and the quantification of the investment needed for its rehabilitation; and (ii) the total cost for comparison of different alternatives of rehabilitation actions.

In a Portuguese $R \& D$ project, five water utilities have defined a set of 16 performance indicators (Table 1) aiming the assessment and prioritization of water supply systems (WSS) or district metering areas (DMA) for rehabilitation. These performance indicators were regarded as of utmost importance and were implemented in a platform allowing its calculation after the integration of the required data (Carriço et al., 2020).

The calculation of these performance indicators relies in a deep knowledge of managed systems and its respective assets. This knowledge is provided by data that is collected and stored in different databases, generally spread in various departments or divisions from the water utility. For instance, pipe failures may come from a service work order register whilst real water losses in network comes from a water balance calculation. Therefore, the infrastructure asset manager has a hard task every time he needs to assess their systems since data must be collected from different stakeholders in a coordinated procedure (Carriço et al., 2020).

\section{DATA REQUIREMENTS FOR CONDITION ASSESSMENT}

The basic data needed to assess urban water infrastructure asset's condition may be categorized into four main groups, namely:

i. Cadastral-detailed information of the system's different assets including identification, type, location, dimensions, shape, material, among others.

ii. Functional and physical condition-studies about the hydraulic behavior such as mathematical modeling and hydraulic measurements, performance assessment reports (assessment period, performance indicators used and targets considered), among others.

iii. Operational and maintenance-including data on asset maintenance activities (e.g., dates, locations), failure reports, data complaints (e.g., low pressure), among others.

iv. Billing and account information-network related maintenance and operational costs, energy and personnel costs, amortizations and interest, revenues, among others.

Data collection thus is an activity that significantly consumes human, technological, and financial resources. However, important data is often neither collected nor recorded, or the available data does not respond to the needs or does not fulfill the requirements for the different stakeholders. In some cases, water utility technicians are required to collect too much data, which in result is simply not registered due to personnel lack of time. For example, the authors have analyzed the service work orders of a few Portuguese water utilities and found out that they usually have c.a. 200 possible fields regarding each work order. Nonetheless, most of these fields (i.e., around 90\%) where simply null as they were left blank. For that reason, the water utility should firstly identify the necessary information requirements to fulfill its objectives (in which one is asset's condition assessment) in order to rationalize the use of resources and maximize data collection effectiveness.

These data are then stored and used in the water utility's activities using several IS namely, Geographic Information System (GIS), Customer Relationship Management (CRM), Costumer Information System (CIS), Enterprise Resource Planning system (ERP), and Supervisory Control and Data Acquisition System (SCADA), Computerized Maintenance 


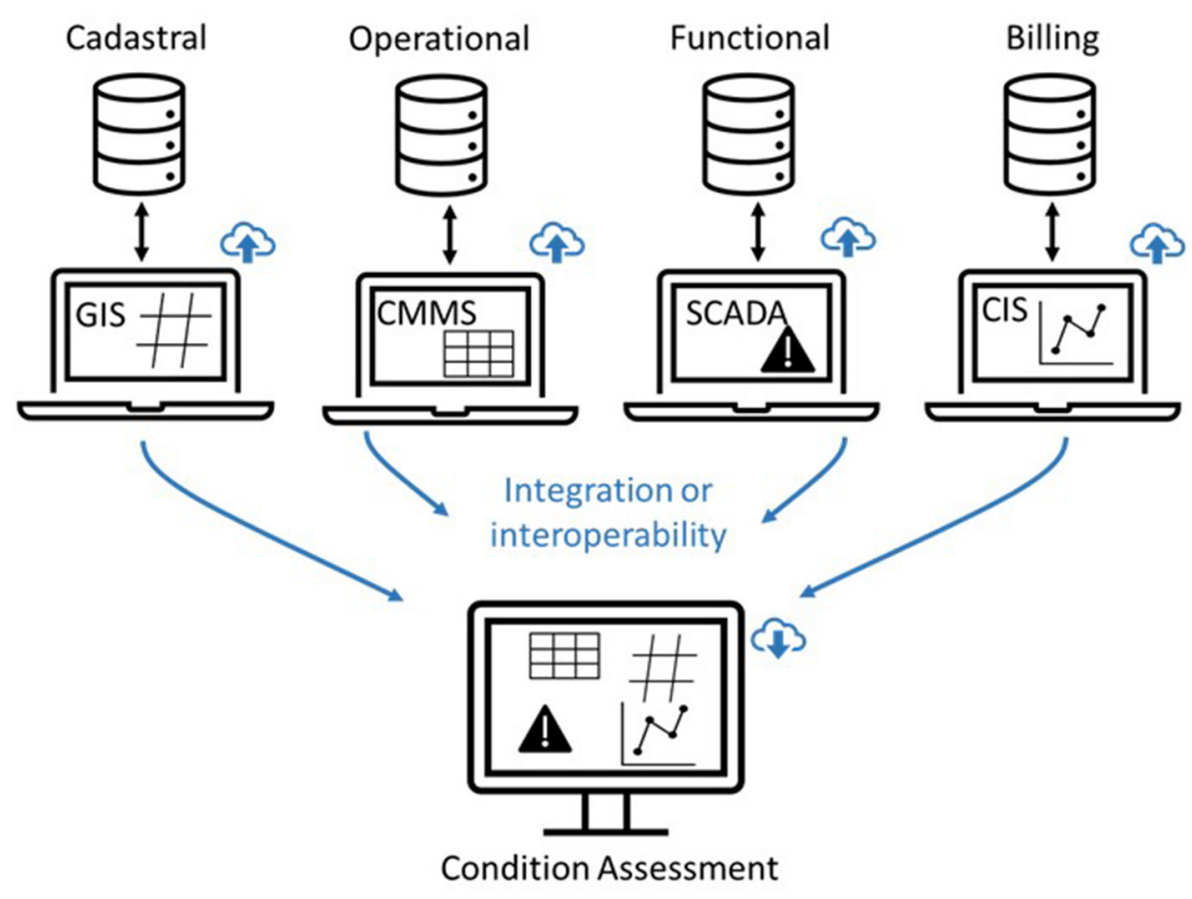

FIGURE 1 | Data requirements and information fluxes for condition assessment.

Management System (CMMS), Laboratory Information Management System (LIMS), among others. Figure 1 depicts a possible scheme for the data requirements and information fluxes for condition assessment.

As the large amount of assets and data involved makes data and information management a challenging task, new tools, and processes are often needed to collect, gather, manage, analyse, and use asset data. Creating and using these tools can stimulate and improve knowledge within organization and decision making, ultimately improving utilities' information management (Alegre et al., 2015).

\section{DATA INTEGRATION AND INTEROPERABILITY}

The assessment of asset's condition is carried by crossing data and information from the different IS existing in the utility. A way to put data and information together is by integration and/or interoperability. Data integration refers to the connection of different IS so that data from one system can be accessed by another. As such, data integration usually involves a third software called middleware that translates the data and makes it readable for the receiving system. Data integration is important to enable cross functional processes as well as provides quality analysis of asset condition assessment. Interoperability refers to the ability of an IS to connect and communicate with another IS, even if they were created by different developers. When systems are interoperable, they can not only share information, but interpret the data received and present it in its original form. Interoperability not only brings together various systems but also contributes to functional and organizational integration (Haider, 2013). Figure 2 exemplifies data interoperability and integration in the context of condition assessment.

Water utilities with a greater digitalization maturity level may have the required resources to develop their own data integration/interoperability solutions. These solutions integrate data gathered from different sources (i.e., sensors, GIS, equipment automation signals, among others), as well as results from hydraulic simulation models, aiming ultimately at predictive models of network hydraulic behavior and decision support tools (Vieira et al., 2020). Some water utilities choose to integrate data through GIS driven solution, in which it is possible to easily access data related to infrastructures, fleets, water demands, billing, among others (Silva et al., 2017; Sousa et al., 2017). Nonetheless, the use of these solutions is restricted to the utility itself and most water utilities do not dispose the capacity to develop their own solutions. Different commercial software (e.g., Baseform, WaterSmart) claim to provide comprehensive, actionable analytics to assist water utilities specific efficiency issues and key business outcomes (Carriço et al., 2020). However, the cost of such solutions is usually a barrier to many water utilities.

\section{DATA AND INFORMATION MANAGEMENT BARRIERS AND RECOMMENDATIONS}

The information management efficiency of water utilities often falls short of desired results. This is particularly 

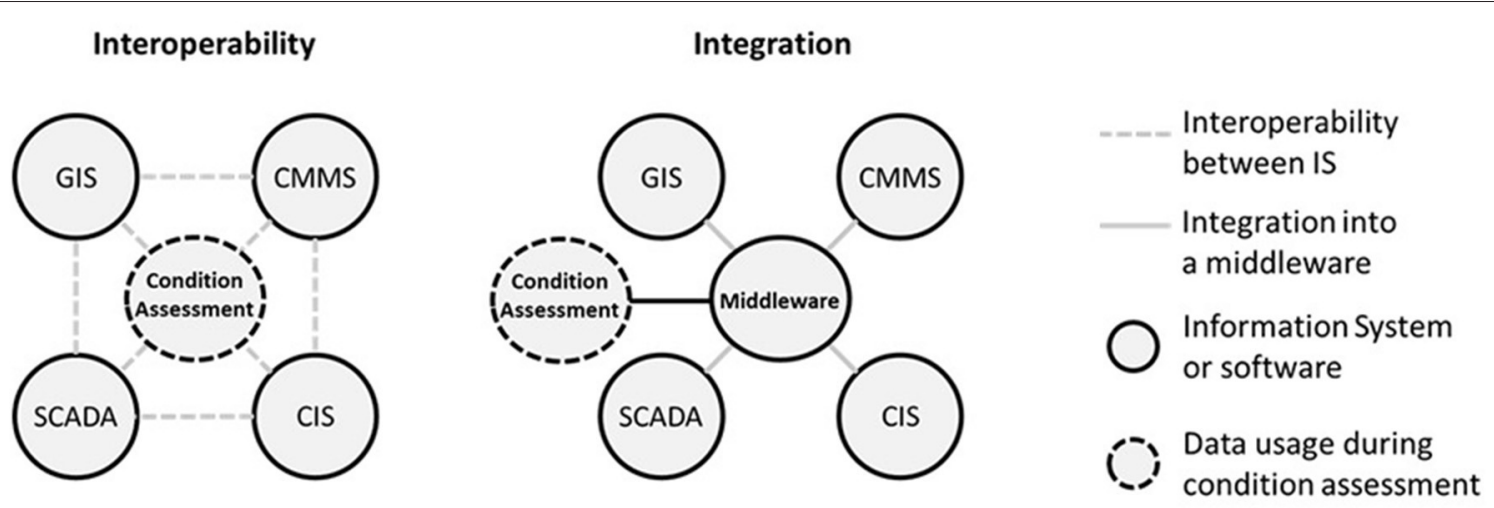

FIGURE 2 | Data integration and interoperability in the context of condition assessment.

prominent in municipalities since management is usually structured according to local government models. Due to lack of technical capacity and human resources, it is not usually possible to generate the critical information through daily routines (e.g., well-planned, and organized service orders that can ensure useful, good-quality information). The most frequent problems in information management found by the authors in Portuguese water utilities include:

- Great diversity of existing IS.

- Lack of IS integration and/or interoperability.

- Existence of several legacy systems that require updating or replacement.

- Strong competition between information management systems.

- Lack of strategic vision on the technological environment of the water utility.

- Limited and irregular use by utility workers.

- Poor data quality, including lack of consistency, duplication, and outdatedness.

- Lack of support and recognition from the management of the water utility for the importance of information management.

- Limited resources to deploy, manage, or improve IS.

- Difficulty in altering workers' processes and workflows.

- Lack of coordination or communication between the various users of information within the utility.

There are several practices and solutions that may reduce or limit the listed problems, namely:

- Standardization of the data stored in the different IS in order to make them compatible with each other.

- Improvement of the quality of the collected data by periodically reviewing the data model, eliminating duplicated data, and cleaning of the outdated data.

- Optimization of information processes and flows.

- Guarantee the protection and security of data using IS in accordance with the General Data Protection Regulation (GDPR) and other data protection regulations.
- Creation of a data recovery strategy, as it is common to both make mistakes when entering data and to delete data inadvertently.

\section{FUTURE TRENDS}

The fourth industrial revolution has brought more sophisticated solutions to urban water infrastructure asset management, namely:

- Internet of Things (IoT) allows water utilities to have smart water metering that measure and monitor their networks in real-time.

- Artificial intelligence (AI) solutions, that are changing the water industry by leveraging the collected data to create more effective and intelligent water management and operations.

- Augmented and virtual realities (AR/AV) by assisting water utilities in their daily operations and management activities also training by providing better ways of data and information visualization.

- Digital twins, which make it possible to build what-if scenarios and incur suitable outcomes throughout the asset's lifecycle allowing better planning and training activities.

However, these promising technologies are not ready-to-use by the most water utilities, especially in those with lower digitalization maturity level. Notwithstanding the fact that a large sum of capital investment is needed to implement any technology, these water utilities face some barriers that need to be firstly solved. Such barriers often include the lack of integration and interoperability of the existing IS, resistance of the human resources to change their cultural mindset and work habits, lack of infrastructure (e.g., access to wi-fi, power source), cybersecurity, amongst others.

\section{FINAL REMARKS}

The large amount of assets and data involved coupled with the lack of human and financial resources makes data and 
information management a challenging task for water utilities. This challenge is greater in those utilities with a lower digital maturity level. New tools and processes in information systems are being developed, motivated by the growing need to make water utilities more resilient and flexible, with greater transparency and rationality in the decision-making process, as well as to respond to challenges more quickly and efficiently. The development and use of these tools can be a stimulative process to water utilities, further improving the knowledge within the organization and decision-making processes.

However, many small and medium size utilities worldwide are yet resistant to progress to the digital era initiated by the third industrial revolution, which in turn bring difficulties the progress to the automation era initiated by the fourth industrial revolution. These utilities should start to investigate the data they collect and to rethink existing data models. These models should be simplified and standardized between them in order to avoid data duplication and to improve data quality.

\section{REFERENCES}

Alegre, H., Brito, R. S., Neves, C., and Almeida, R. (2015). "Standarization in infrastructure asset management (in Portuguese)," in ENEG Vol. 55000.

Alegre, H., and Coelho, S. T. (2012). "Infrastructure asset management of urban water systems," in Water Supply System Analysis - Selected Topics Ostfeld. Water Supply System Analysis - Selected Topics, ed B. T. Avi (Rijeka: InTech), 1-26.

Alegre, H., and Covas, D. (2010). Gestão Patrimonial de Infra-Estruturas de Abastecimento de Água: Uma Abordagem Centrada Na Reabilitação. Série Guias Técnicos, Vol. 16, ed ERSAR. Lisboa: ERSAR.

Almeida, M. C., and Cardoso, M. A. (2010). Infrastructure Asset Management of Wastewater and Stormwater Services. An Aproach Based on Rehabilitation (in Portuguese). Technical Guide n.17. Lisbon: ERSAR; LNEC.

AS/NZS (2004). AS/NZS 4360:2004 - Risk Management. Standards Australia.

AWWA (2019). Condition Assessment of Water Mains - Manual M77. American Water Works Association.

Beuken, R., Eijkman, J., Savic, D., Hummelen, A., Blokker, M., and Eijkman Evides, J. (2020). Twenty years of asset management research for dutch drinking water utilities. Water Supply 20, 2941-2950. doi: 10.2166/ws.2020.179

BSI (2008). PAS 55-1:2008 Asset Management. Specification for the Optimized Management of Physical Assets. The Institute of Asset Management.

Carriço, N., Ferreira, B., Barreira, R., Antunes, A., Grueau, C., and Mendes, A., et al. (2020). Data integration for infrastructure asset management in small to medium-sized water utilities. Water Sci. Technol. 82, 2737-2744. doi: $10.2166 /$ wst.2020.377

Feeney, C., Thayer, S., Bonomo, M., and Martel, K. (2009). White Paper on Condition Assessment of Wastewater Collection Systems. Cincinnati, OH.

Haider, A. (2013). Information Systems for Engineering and Infrastructure Asset Management. Information Systems for Engineering and Infrastructure Asset Management. Gabler Verlag.

Haider, A. (2007). Information Systems for Engineering and Infrastructure Asset Management. Springer Gabler.

Halfawy, M. R. (2008). Integration of municipal infrastructure asset management processes: challenges and solutions. J. Comput. Civil Eng. 22, 216-229. doi: 10.1061/(ASCE)0887-3801(2008)22:3(216)

IAM (2015). Asset Management- An Anatomy. Bristol.

IPWEA and NAMS (2015). International Infrastructure Management Manual, 5th Edn. Sydney, NSW; Wellington: IPWEA; NAMS Group.

\section{AUTHOR CONTRIBUTIONS}

All authors listed have made a substantial, direct and intellectual contribution to the work, and approved it for publication.

\section{FUNDING}

This paper was developed under the WISDom project supported by the Fundação para a Ciência e a Tecnologia (Grant Number: DSAIPA/DS/0089/2018) through the Data Science and Artificial Intelligence in Public Administration Programme.

\section{ACKNOWLEDGMENTS}

The authors acknowledge the Fundação para a Ciência e a Tecnologia for supporting this research, the project's team, and participating water utilities for their contribution.

ISO (2009). ISO Guide 73:2009 - Risk Management - Vocabulary. International Organization for Standardization.

ISO (2014). ISO 55000:2014, Asset Management - Overview, Principles and Terminology. International Organization for Standardization.

ISO (2018). ISO 31000:2018 - Risk Management - Principles and Guidelines. International Organization for Standardization.

Matthews, J. C., Selvakumar, A., Sterling, R., and Condit, W. (2012). Analysis of wastewater and water system renewal decision-making tools and approaches. J. Pipeline Syst. Eng. Prac. 3, 99-105. doi: 10.1061/(ASCE)PS.1949-1204.00 00114

Osman, H. (2012). Agent-based simulation of urban infrastructure asset management activities. Automation Construct. 28, 45-57. doi: 10.1016/j.autcon.2012.06.004

Silva, M., Vilela, J., and Morais, M. (2017). "Integration between information systems. Contribution to the provision of quality service (In Portuguese)," in Proceedings of ENEG 2017 (Évora).

Sousa, C., Marques, A., and Fernandes, J. (2017). "The contribution of GIS information for an integrated and efficient management systems (In Portuguese)," in Proceedings of ENEG 2017 (Évora).

Ugarelli, R., Venkatesh, G., Brattebo, H., Di Federico, V., and Saegrov, S. (2010). Asset management for urban wastewater pipeline networks. J. Infrastruct. Syst. 16, 112-121. doi: 10.1061/(ASCE)IS.1943-555X. 0000011

Vieira, P., Costa, F., Cunha, R., Marques, R., and Fernandes, M. (2020). "Technological platform for the urban water cycle. From concept to implementation (In Portuguese)," in Proceedings of 18th ENASB (Porto).

WRc (2001). Sewer Rehabiltation Manual. Water Research Council Publications.

Conflict of Interest: The authors declare that the research was conducted in the absence of any commercial or financial relationships that could be construed as a potential conflict of interest.

Copyright (C) 2021 Carriço and Ferreira. This is an open-access article distributed under the terms of the Creative Commons Attribution License (CC BY). The use, distribution or reproduction in other forums is permitted, provided the original author(s) and the copyright owner(s) are credited and that the original publication in this journal is cited, in accordance with accepted academic practice. No use, distribution or reproduction is permitted which does not comply with these terms. 